\title{
Parameter Optimization of UWB SRR System Performance in Weibull Clutter Environment
}

\author{
Purushothaman Surendran ${ }^{1}$, Jong-Hun Lee ${ }^{2}$ and Seok Jun Ko ${ }^{1}$ \\ ${ }^{1}$ Department of Electronics Engineering, Jeju National University, Jeju, Korea \\ ${ }^{2}$ Daegu Gyeongbuk Institute of Science \& Technology, Daegu, Korea \\ sjko@jejunu.ac.kr
}

\begin{abstract}
The objective of this paper is to optimize the parameters of non-coherent detectors such as coherent and non-coherent integration number for various non-coherent detectors such as square law detector, linear detector and logarithmic detector in weibull clutter environment for Ultra Wide Band Short Range Radar in Automotive applications. The detection performance of the detectors is analyzed for fixed false alarm probability of 0.001 and simulation has been done in order to verify it.
\end{abstract}

Keywords: UWB SRR, Coherent Integration, Non-coherent integration, Weibull Clutter

\section{Introduction}

UWB Automotive Radar technology is a key enabling technology for innovative driver assistance systems and safety systems. Intelligent Transportation System uses UWB SRR system for driver's safety and convenience. Ultra wideband (UWB) offers many applications to vehicle such as pre-crash warning system, stop and go operation, spot assist and lane change assist. Clutter is generally distributed in spatial extent in that it is much larger in physical size than the radar resolution cell [1]. The ability to determine range by measuring the time for the radar signal to propagate to the target and back is probably the distinguishing and most important characteristic of radar. In order to suppress the clutter, a pulse integration and Constant False Alarm Rate (CFAR) has been employed. It is therefore important to investigate the more appropriate clutter model to resemble the clutters in automobile applications. In high resolution radars, the $\log$ normal and weibull clutters are proved to be better suited for clutter model in automobile applications. The road clutter resembles weibull distribution for $100 \mathrm{MHz}$ bandwidth.

The frequency band of $21.625-26.625 \mathrm{GHz}$ is allocated for the use of UWB short range radar in automotive applications [1-2]. Sea clutter measured by means of rowresolution radar on $\mathrm{L}$ and $\mathrm{X}$-band has been studied and reported [3]. Recently, ground clutter data has been measured by Ka-band radar [4].

In this paper, we optimize the non-coherent detector parameters such as coherent integration and non-coherent integration. Finally, we analyze the detection performance of non-coherent detectors such as square law detector, linear detector and logarithmic detector in weibull clutter environment for fixed false alarm probability of 0.001 by using Monte Carlo simulation. 
The organization of this paper is as follows. In Section II, the non-coherent detectors are described. In Section III, the simulation results are shown. Finally, conclusion is presented in Section IV

\section{UWB SRR Detector}

In this section, we present the non-coherent detector which consists of coherent integrator and non-coherent integrator. The coherent and non-coherent range gate memory size $(K)$ is less than the maximum range and indicates the total number of target range to be tested. These range gates are used as buffer to integrate the values coherently and non-coherently. Therefore, at every $T_{P R I}$, we use the samples as much as the range gate's memory size $(K)$.

The sampling frequency of ADC is same to the pulse width $\left(T_{P}\right)$. Thus each sampled values of the in-phase (I) and quadrature (Q) channel are shifted at every $T_{P}$ during one $T_{P R I}$. At every $T_{P R I}$, we use the samples only as much as the range gate's memory size $(K)$. The coherent integrator integrates the sample values at each range gate during $N_{c} \cdot T_{P R I}$ (where $N_{c}$ is the number of coherent integration). If the round trip delay $(\tau)$ from target is equal to the time position of the $k$-th range gate $\left(k \cdot T_{P}\right)$, then the target maximum range can be expressed as $k \cdot T_{P} / 2=k \cdot \Delta R$. And then the range resolution is given by $\Delta R=c \cdot T_{P} / 2$.

The transmitted signal can be written as follows

$$
s(t)=A_{T} \cdot \sin \left(2 \pi f_{c} t+\varphi_{0}\right) \cdot \sum_{n=-\infty}^{+\infty} p_{n}(t)
$$

where $p_{n}(t)$ is the Gaussian pulse train; $A_{T}$ is the amplitude of single transmit pulse, $\varphi_{0}$ is the phase of the transmit signal, $f_{c}$ is the carrier frequency [11].

In this paper, we assume one reflected signal from single stationary target against a background of weibull clutter environment, and then the baseband complex received signal can be described as

$$
\bar{r}(t)=A_{T} \sum_{n=-\infty}^{+\infty} \alpha \cdot e^{j\left(\omega_{d} t+\theta\right)} p\left(t-n T_{P R I}-\tau\right)+\bar{C}(t)
$$

where the amplitude of the scatter $\alpha$, the time delays of the scatters $\tau$ and the arbitrary phase $\theta=-2 \pi f_{c} \tau+\varphi_{0}$ are all unknown. Also, $\bar{C}(t)$ is the reflected clutter signal from unwanted object. Doppler shift is denoted as $\omega_{d}= \pm 4 \pi v / \lambda= \pm 4 \pi v f_{c} / c$ where the wavelength $\lambda$ is $c / f_{c}$ and $c$ is the velocity of light. In the Doppler shift, the positive sign $(+)$ indicates the closing target and the negative sign (-) means the receding target.

The output of the coherent integrator can be distinguished in to two hypotheses,

$$
\begin{aligned}
H_{1} & =\overline{X_{k}}(m)=\frac{A_{T} \alpha}{N_{c}} \sum_{n=m N_{c}}^{(m+1) N_{c}-1} e^{j\left(\omega_{d} t+\theta\right)} p\left(t-n T_{P R I}-\tau\right)+\bar{C}(n), \\
H_{0} & =\overline{X_{k}}(m)=\frac{1}{N_{c}} \sum_{n=m N_{c}}^{(m+1) N_{c}-1} \bar{C}(n),
\end{aligned}
$$

where $m$ indicates the $m$-th coherent integration and $H_{I}$ is for $\tau=k \cdot T_{P}$ and $H_{0}$ is for $\tau \neq k \cdot T_{P}$. Also, the sampling rate of the ADC is equal to the pulse width. The baseband 
received signal is sampled at peak point of $p(t)$. Therefore, we get the coherently integrated values as $\left(\overline{X_{k}}(m), k=1,2, \ldots, K\right)$. The sample value received from the coherent integration is squared and operates at every $N_{c} \cdot T_{P R I}$. The squared range gate samples are combined and then both I and Q branch values are summed as shown in Figure 2(a).

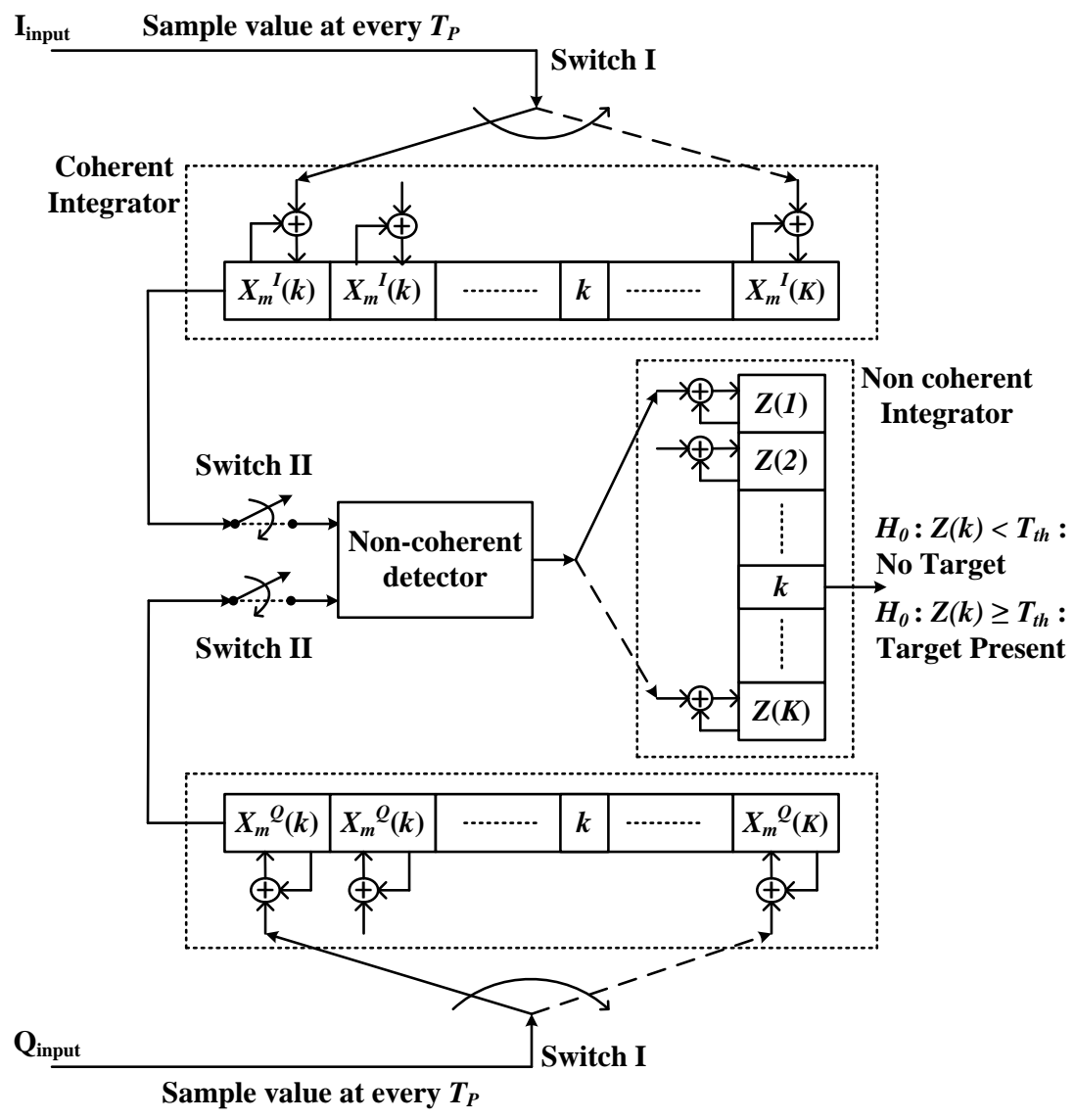

Figure 1. Block Diagram of receiver

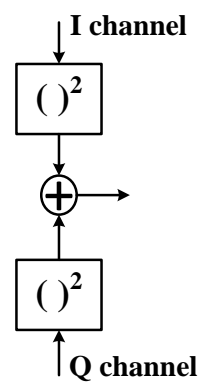

(a) Square law

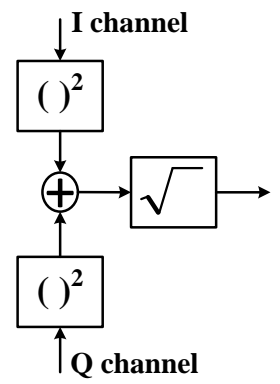

(b) Linear

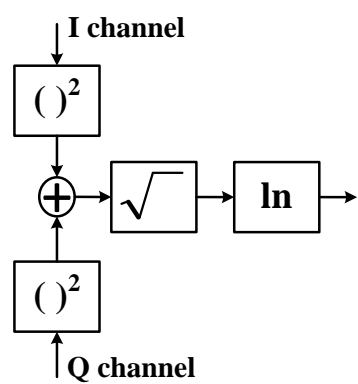

(c) Logarithmic

Figure 2. Non-coherent Detector 
The $k$-th range gate value after squaring can be represented as

$$
Y_{k}(m)=\left\{X_{k}^{I}(m)\right\}^{2}+\left\{X_{k}^{Q}(m)\right\}^{2} .
$$

In the case of linear detector the squared range gate samples are combined and square root is applied to the combined values as shown in Figure 2(b). The output of the linear detector can be represented as

$$
Y_{k}(m)=\sqrt{\left\{X_{k}^{I}(m)\right\}^{2}+\left\{X_{k}^{Q}(m)\right\}^{2}} .
$$

In the case of logarithmic detector the squared range gate samples are summed, square root and natural logarithmic is applied to the combined values as shown in Figure 2(c). The output of the linear detector can be represented as

$$
Y_{k}(m)=\ln \left(\sqrt{\left\{X_{k}^{I}(m)\right\}^{2}+\left\{X_{k}^{Q}(m)\right\}^{2}}\right) \text {. }
$$

The value $Y_{k}(m)$ is stored in the $k$-th memory of the non-coherent integration at every $N_{c} \cdot T_{P R I}$. The output of the non-coherent integration $Z(k)$ can be written as

$$
Z(k)=\frac{1}{N_{n c}} \sum_{l=1}^{N_{n c}} Y_{k}(m)
$$

If the above result is greater than the defined threshold, then we can determine that a target is present. And the index $k$ represents the position of the target; the target range indicates $k \cdot 30 \mathrm{~cm}$. It takes $N_{c} \cdot N_{n c} \cdot T_{P R I}$ to decide the target range.

\section{Simulation Result}

The purpose of the simulation is to assess the detection performance of the noncoherent detectors in weibull clutter environment. The various optimized parameters used in the simulation are as follows; the coherent integration number $\left(N_{c}\right)$ and the noncoherent integration number $\left(N_{n c}\right)$ [10]. The percentage of total energy reflected from the target is assumed to be 1 . The signal-to-clutter ratio (SCR) is defined as $\bar{E} / \mathrm{C}_{0}$, where $\overline{\mathrm{E}}$ represents the total average energy reflected from a target.

The weibull distribution can be expressed as follows

$$
p(x)=\frac{\alpha}{b}\left(\frac{x}{b}\right)^{\alpha-1} \exp \left[-\left(\frac{x}{b}\right)^{\alpha}\right],
$$

where $b$ is the scale parameter $(b>0), \alpha$ is the shape parameter $(\alpha>0)$ and $x$ is the random variable of the weibull distribution.

The clutter power $\left(\mathrm{C}_{0}\right)$ for weibull environment can be expressed as follows

$$
C_{0}=\frac{x_{m}^{2} \Gamma(1+(2 / \alpha))}{(\ln (2))^{2 / \alpha}}
$$


where $\alpha$ is the shape parameter and indicates the degree of skewness, $x_{m}$ is the median of weibull distribution [8]. From Figure 3, we can predict that the clutter power is approximately $1 \mathrm{~dB}$ for shape parameter greater than 3 . Also, we know that the weibull scale and shape parameter for $24 \mathrm{GHz}$ automobile short range radar is 1.6 and 6.9 for traffic road clutter [2]. Thus, we use the maximum scale and shape parameter value as 2 and 7 in the simulation.

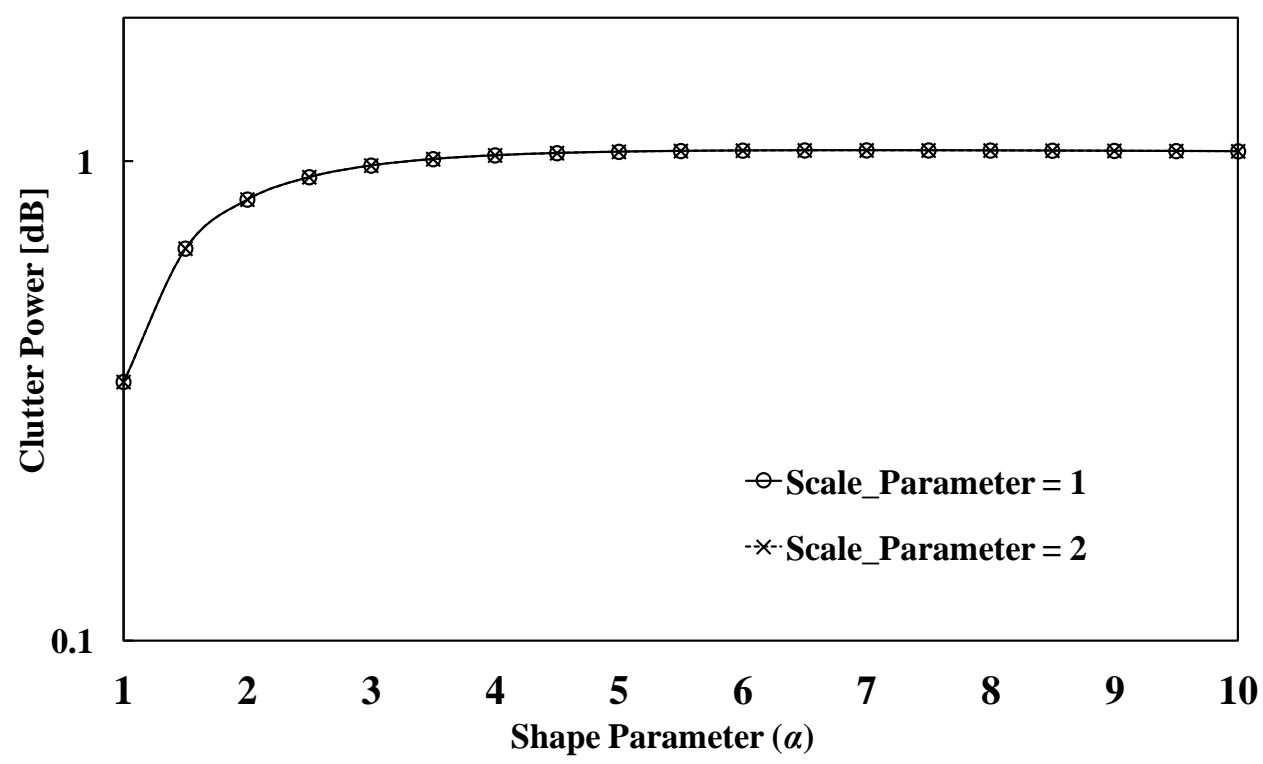

Figure 3. Weibull Clutter power for various scale parameters

Figure 4 and 5 shows the detection probability versus weibull skewness parameter $(\alpha)$ at $P_{F A}=0.001$. The simulation result shows that the probability of detection is approximately 1 for non-coherent integration number of 16 , for skewness $(\alpha)$ and scale parameter $(b)$ greater than 4 and 2. Also, we can predict that the detection probability increases as the non-coherent integration number increases for fixed false alarm probability. Finally, from Figure 5 the detection performance of logarithmic detectors is good for skewness values less than 4 . Thus, in this paper the coherent and non-coherent integration number of 10 and 16 is optimized for false alarm probability of 0.001 in weibull clutter environment. Finally, the detection probability of non-coherent detectors is almost 1 for skewness parameter greater than 4 and optimized non-coherent detector parameters in weibull clutter environment. 


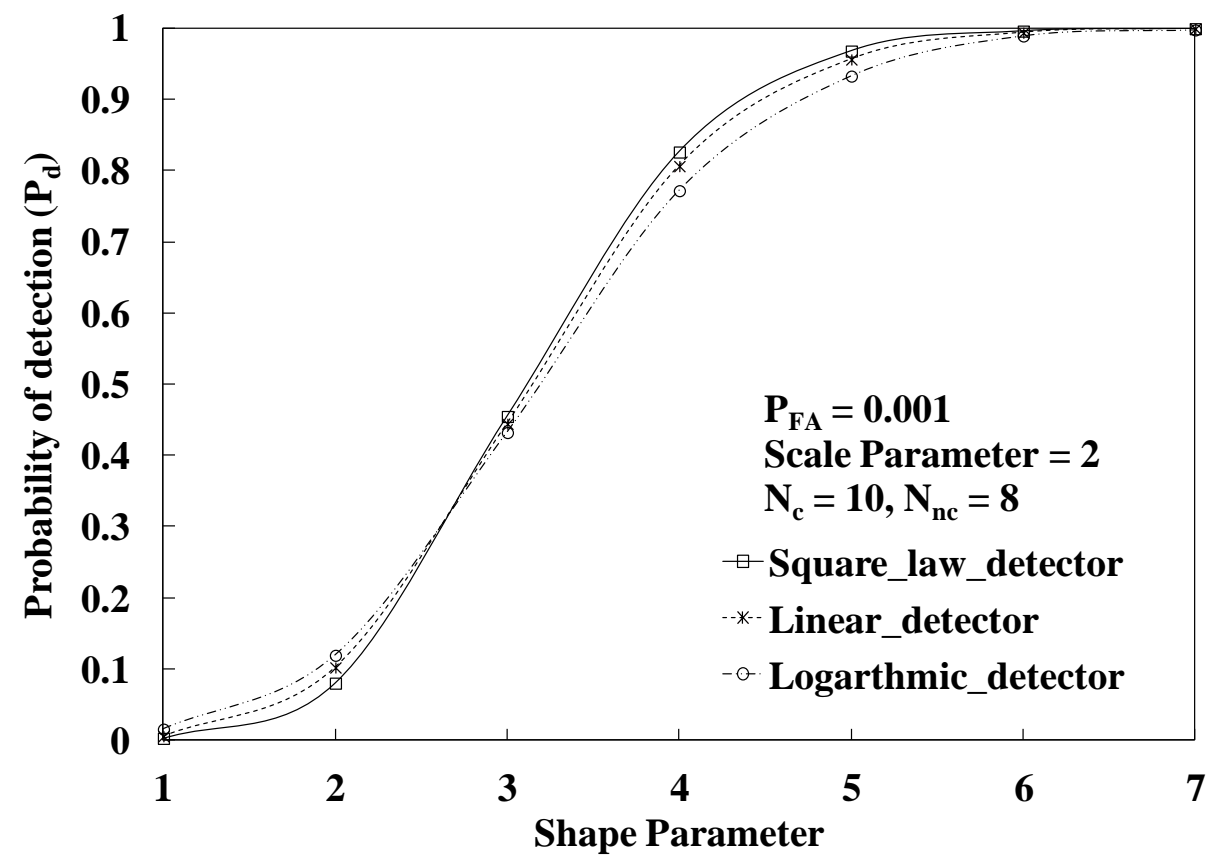

Figure 4. Shape Parameter Vs Probability of detection

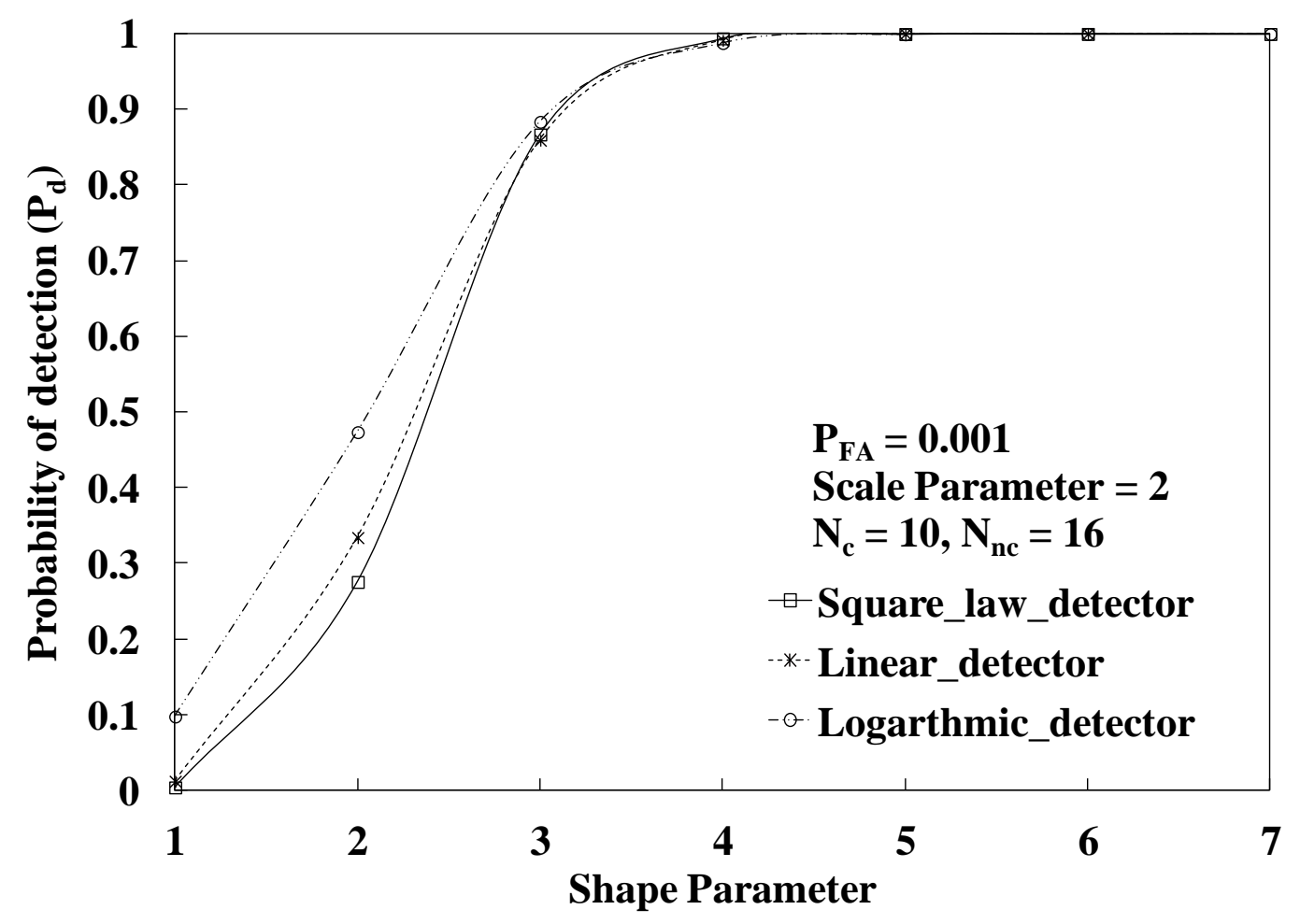

Figure 5. Shape Parameter Vs Probability of detection 


\section{Conclusion}

In this paper, we have analyzed the detection performance of non-coherent detectors for UWB-SRR in automotive applications. The detection probability is found to be same for the non-coherent detectors such as square law detector, linear detector and logarithmic detector for skewness parameters greater than 4. Finally, in order to get the detection probability of more than 0.9 for $P_{F A}=0.001$, the coherent and non-coherent integration number of approximately 10 and 16 is required for non-coherent detectors in weibull clutter environment.

\section{Acknowledgements}

This work was supported by the DGIST R\&D program of the ministry of education \& science technology, Korea (12-RS-02).

\section{References}

[1] M. I. Skolnik, "Introduction to radar systems", Third Edition: McGraw-Hill, (2001).

[2] I. Matsunami, Y. Nakahata, K. Ono, Y. Noguchi and A. Kajiwara, "Empirical Study on Ultra-Wideband", Vehicular Technology conference hall, (2008).

[3] S. Sayama and M. Sekine, "Observations and suppression of sea ice clutter by means of millimeter wave radar", IEICE Trans Communications (Japanese Edition), vol. J86-B, no. 7, (2002) July, pp. 1104-1111.

[4] S. Sayama and M. Sekine, "Amplitude statistics of ground clutter included town using millimeter wave radar”, IEICE Trans. Communications (Japanese Edition), vol. J86-B, no. 5, (2003) May, pp. 829-836.

[5] K. M. Strohm, "Development of Future Short Range Radar Technology", Radar Conference, (2005).

[6] J. D. Taylor, "Ultra-Wideband Radars Technology: Main Features Ultra-Wideband (UWB) Radars and differences from common Narrowband Radars", CRC Press, (2001).

[7] M. Klitz, “An Automotive Short Range High Resolution Pulse Radar Network”, Ph. D. Dissertation, (2002) January.

[8] G. Minkler and J. Minkler, “The Principles of Automatic Radar Detection in Clutter”, CFAR, Magellan Book Company, (1990).

[9] J. G. Proakis, "Digital Communications", McGraw-Hill, (2001).

[10] P. Surendran, J. -H. Lee and S. -J. Ko, "Performance of UWB Short Range Radar in Weibull Clutter Environment", European Radar Conference, (2012).

[11] P. Surendran, S. -J. Ko, S. -D. Kim and J. -H. Lee, "A Novel Detection Algorithm for Ultra Wide Band Short Range Radar in Automobile Application”, IEEE VTC2010-Spring, (2010) May.

\section{Authors}

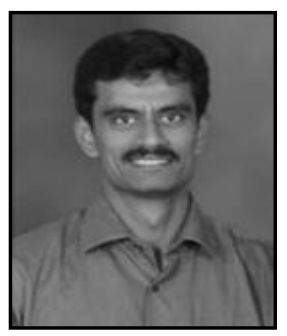

\section{Purushothaman Surendran}

He received the B.E degree in Electronics and communication engineering from Anna University, India in 2005 and M.E. degree in embedded systems from Coimbatore Institute of Technology, India in 2007, respectively. During 2007-2008, he stayed in VIT University, India. Since 2009, he is with Department of Electronics Engineering, Jeju National University, Korea as a Ph.D student. His research interest is UWB radar and Signal Processing. 


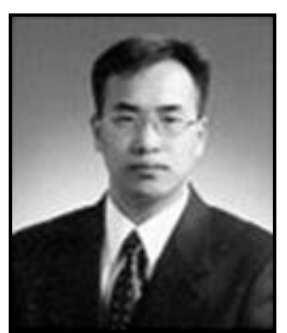

\section{Jong-Hun Lee}

He received the B.S., M.S. and Ph.D degree in electronics engineering from SungKyunKwan University, Korea, in 1996, 1998, 2002, respectively. From 2002 to 2005, he joined Samsung Electronics Company as a Senior Research engineer. Since 2005, he has joined in the DGIST, Korea, as a senior research engineer. His primary research interests are the detection, tracking, recognition for radar (FMCW \& UWB radar) and vision-based vehicle sensor.

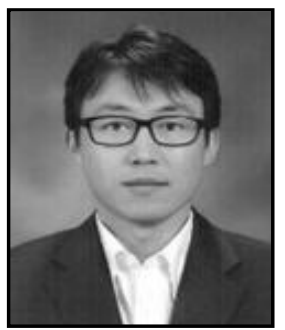

\section{Seok-jun Ko}

He received the B.S., M.S., and Ph. D. degrees in Electronics Engineering from SungKyunKwan University of Korea in 1996, 1998, and 2001, respectively. During 2002-2004, he stayed in Samsung Electronics Inc. He is now with Department of Electronics Engineering, Jeju National Univ., Korea. His interests are the signal processing in communication and radar systems. 